\title{
Existence and data dependence results for fractional differential equations involving atangana-baleanu derivative
}

\author{
Sagar T. Sutar ${ }^{1} \cdot$ Kishor D. Kucche $^{2}$ (D) \\ Received: 29 August 2020 / Accepted: 19 May 2021 / Published online: 17 June 2021 \\ (c) The Author(s), under exclusive licence to Springer-Verlag Italia S.r.l., part of Springer Nature 2021
}

\begin{abstract}
In the current paper, we consider multi-derivative nonlinear fractional differential equations involving Atangana-Baleanu fractional derivative. We investigate the fundamental results about the existence, uniqueness, boundedness and dependence of the solution on various data. The analysis is based on a fractional integral operator due to T. R. Prabhakar involving generalized Mittag-Leffler function, the Krasnoselskii's fixed point theorem and Gronwall-Bellman inequality with continuous functions.
\end{abstract}

Keywords Multi-derivative fractional differential equations $\cdot$ Atangana-Baleanu derivative $\cdot$ Existence and uniqueness · Dependence of solution · Gronwall-Bellman inequality

Mathematics Subject Classification $26 \mathrm{~A} 33 \cdot 34 \mathrm{~A} 12 \cdot 34 \mathrm{~A} 08 \cdot 34 \mathrm{~A} 40$

\section{Introduction}

Fractional differential equations (FDEs) [1-5] appeared as an excellent mathematical tool for modeling of many physical phenomena appearing in various branches of science and engineering such as viscoelasticity, self-similar protein dynamics, continuum and statistical mechanics, dynamics of particles etc. For more details, one can refer [6-11] and furthermore articles referred in that. Crucial development about existence and uniqueness theory, various sorts of stabilities, data dependency and the controllability results for a different class of FDEs can be found in [12-16] and the references cited therein.

To avoid the singularity appearing in the classical fractional differential operators many researchers are attempting to build up the theory of fractional calculus by constructing different kinds of fractional derivative operators with the nonsingular kernel. In this sense,

Kishor D. Kucche

kdkucche@gmail.com

Sagar T. Sutar

sutar.sagar007@gmail.com

1 Department of Mathematics, Vivekanand College (Autonomous), Kolhapur 416003, Maharashtra, India

2 Department of Mathematics, Shivaji University, Kolhapur 416 004, Maharashtra, India 
Caputo and Fabrizio [17] constructed a new fractional derivative which a variant of Caputo derivative with the singular kernel replaced by the exponential function as its kernel. Atangana and Baleanu in [18] introduced non singular Caputo and Riemann-Liouville version of fractional differential operator with Mittag-Leffler function as its kernel.

Taking advantage of the non-singular Mittag Leffler kernel present in the AtanganaBaleanu (AB)-fractional derivative operators, recently authors from various branches of applied mathematics developed and studied mathematical models involving AB-fractional derivative. Bonyah et al. [19] considered a mathematical model involving AB-fractional derivative for co-infection of cancer and hepatitis diseases. They examined existence and uniqueness, stability analysis, and reproductive number. In [20], Ahmad et al. investigated the fractional-order tumor-immune-vitamin model with AB-fractional derivative for existence, uniqueness, and Hyres-Ulam stability. Authors in [21] did a comparative and chaotic study of tumor and effector cells through the fractional tumor-immune dynamical model with $\mathrm{AB}$-fractional derivative. In [22], the authors utilized the fractional $\mathrm{AB}$ derivative to study the numerical solution of the fractional immunogenetic tumor model. Study of transmission dynamics of COVID-19 mathematical model under ABC-fractional-order derivative has been dealt in [23]. Logeswari et al. [24] explored the mathematical model for spreading of COVID-19 infection in the world with AB-fractional derivative. Further, they created a framework that generates numerical outcomes to predict the outcome of the infection spreading all over India. Other few important works that attempted to handle the issue of diverse ailment modeled in the form of FDEs involving AB-fractional derivative are [25-30]. For additional point by point concentrates on various qualitative and quantitative properties of solutions to FDEs with $\mathrm{AB}$-fractional derivative, the interested reader can refer to [31-39].

On the other hand, Mohamed et al. [40], considered multi-derivative initial value problem for Caputo FDEs and studied the existence and uniqueness of the solution and obtained numerical solution through Adomian, Picard and predictor-corrector technique. Kucche et al. in [41] extended the work of [40] to the system of multi-derivative FDEs involving the Caputo fractional derivative and studied existence, uniqueness and continuous dependence of solution. Further, they have discussed validity, convergence, and error estimation for Picard's method.

Inspired by the work of [42-44], on the line of [40, 41], we consider multi-derivative nonlinear FDEs involving Riemann-Liouville version of AB-fractional derivative (ABR derivative) of the from:

$$
\begin{gathered}
\frac{d \omega}{d \tau}+{ }_{0}^{*} \mathcal{D}_{\tau}^{\alpha} \omega(\tau)=f(\tau, \omega(\tau)), \tau \in J, \\
\omega(0)=\omega_{0} \in \mathbb{R},
\end{gathered}
$$

where $J=[0, T], T>0,0<\alpha<1,{ }_{0}^{*} \mathcal{D}_{\tau}^{\alpha}$ denotes the ABR-fractional differential operator of order $\alpha$ and $f \in C(J \times \mathbb{R}, \mathbb{R})$ is a non-linear function.

We derive an equivalent fractional integral equation to ABR-FDEs (1.1)-(1.2) analytically and via Laplace transform. Using the properties of fractional integral operator $\mathcal{E}_{\rho, \mu, \omega ; a+}^{\gamma}$, we derive some supplementary results. The existence of solution is obtained by using Krasnoselskii's fixed point theorem. We obtain uniqueness of solution via GronwallBellman inequality as well as using the properties of fractional integral operator $\mathcal{E}_{\rho, \mu, \omega ; a+}^{\gamma}$ The boundedness and the continuous dependence of the solution is obtained through Gronwall-Bellman inequality for continuous function. 
We organize our work as follows: In sect. 2, we recall basic definitions and results about AB-fractional derivative and the generalised Mittag-Leffler function. In sect. 3, we derive an equivalent fractional integral equation to ABR-FDEs (1.1)-(1.2) analytically as well as using the Laplace transform. In sect. 4, we derive supplementary results and existence and uniqueness of solution. In sect. 5, we derive boundedness and data dependence of solution. In sect. 6, an example is provided to illustrate the existence results.

\section{Preliminaries}

In this section, we recall basic definitions and results about $\mathrm{AB}$-fractional derivative and the generalised Mittag-Leffler function.

Definition 2.1 [31] Let $p \in[1, \infty)$ and $\Omega$ be an open subset of $\mathbb{R}$, the Sobolev space $H^{p}(\Omega)$ is defined by

$$
H^{p}(\Omega)=\left\{f \in L^{2}(\Omega): D^{\beta} f \in L^{2}(\Omega), \text { for all }|\beta| \leq p\right\} .
$$

Definition 2.2 [18] Let $\omega \in H^{1}(0,1)$ and $0<\alpha<1$. Then, the left Atangana-Baleanu fractional derivative of $\omega$ of order $\alpha$ in Riemann-Liouville sense (ABR derivative) is defined by

$$
{ }_{0}^{*} \mathcal{D}_{\tau}^{\alpha} \omega(\tau)=\frac{B(\alpha)}{1-\alpha} \frac{d}{d \tau} \int_{0}^{\tau} \mathbb{E}_{\alpha}\left[-\frac{\alpha}{1-\alpha}(\tau-\sigma)^{\alpha}\right] \omega(\sigma) d \sigma,
$$

where $B(\alpha)>0$ is a normalization function satisfying $B(0)=B(1)=1$ and $\mathbb{E}_{\alpha}$ is one parameter Mittag-Leffler function.

Definition 2.3 [18] Let $\omega \in H^{1}(0,1)$ and $0<\alpha<1$. Then, the left Atangana-Baleanu fractional derivative of $\omega$ of order $\alpha$ in Caputo sense is defined by

$$
{ }_{0}^{A B C} \mathcal{D}_{\tau}^{\alpha} \omega(\tau)=\frac{B(\alpha)}{1-\alpha} \int_{0}^{\tau} \mathbb{E}_{\alpha}\left[-\frac{\alpha}{1-\alpha}(\tau-\sigma)^{\alpha}\right] \omega^{\prime}(\sigma) d \sigma,
$$

where $B(\alpha)>0$ is a normalization function satisfying $B(0)=B(1)=1$ and $\mathbb{E}_{\alpha}$ is one parameter Mittag-Leffler function.

Definition $2.4[45,46]$ The generalized Mittag-Leffler function $\mathbb{E}_{\alpha, \beta}^{\gamma}(z)$ for the complex $\alpha, \beta, \gamma$ with $\operatorname{Re}(\alpha)>0$ is defined by

$$
\mathbb{E}_{\alpha, \beta}^{\gamma}(z)=\sum_{k=0}^{\infty} \frac{(\gamma)_{k}}{\Gamma(\alpha k+\beta)} \frac{z^{k}}{k !},
$$

where $(\gamma)_{k}$ is the Pochhammer symbol given by

$$
(\gamma)_{0}=1,(\gamma)_{k}=\gamma(\gamma+1) \cdots(\gamma+k-1), k=1,2, \cdots .
$$

Note that

$$
\mathbb{E}_{\alpha, \beta}^{1}(z)=\mathbb{E}_{\alpha, \beta}(z), \mathbb{E}_{\alpha, 1}^{1}(z)=\mathbb{E}_{\alpha}(z)
$$

We need the following results related with Laplace transformation. 
Lemma 2.1 [18] If $\mathcal{L}\{f(\tau) ; p\}=\bar{F}(p)$, then $\mathcal{L}\left\{{ }_{0}^{*} \mathcal{D}_{\tau}^{\alpha} f(\tau) ; p\right\}=\frac{B(\alpha)}{1-\alpha} \frac{p^{\alpha} \bar{F}(p)}{p^{\alpha}+\frac{\alpha}{1-\alpha}}$.

Lemma $2.2[2] \mathcal{L}\left[\tau^{k \alpha+\beta-1} \mathbb{E}_{\alpha, \beta}^{(k)}\left( \pm a \tau^{\alpha}\right) ; p\right]=\frac{k ! p^{\alpha-\beta}}{\left(p^{\alpha} \mp a\right)^{k+1}}, \mathbb{E}^{(k)}(\tau)=\frac{d^{k}}{d \tau^{k}} \mathbb{E}(\tau)$.

Definition $2.5[46,47]$ Let $\rho, \mu, \omega, \gamma \in \mathbb{C}(\operatorname{Re}(\rho), \operatorname{Re}(\mu)>0), b>a$. Then, the fractional integral operator $\mathcal{E}_{\rho, \mu, \omega ; a+}^{\gamma}$ on a class $L(a, b)$ is defined by

$$
\left(\mathcal{E}_{\rho, \mu, \omega ; a+}^{\gamma} \phi\right)(\tau)=\int_{a}^{\tau}(\tau-\sigma)^{\gamma-1} \mathbb{E}_{\rho, \mu}^{\gamma}\left[\omega(\tau-\sigma)^{\rho}\right] \phi(\sigma) d \sigma, \tau \in[a, b] .
$$

Lemma $2.3[46,47]$ Let $\rho, \mu, \omega, \gamma \in \mathbb{C}(\operatorname{Re}(\rho), \operatorname{Re}(\mu)>0), b>a$, then the operator $\mathcal{E}_{\rho, \mu, \omega ; a+}^{\gamma}$ is bounded on $C[a, b]$ such that

$$
\left\|\left(\mathcal{E}_{\rho, \mu, \omega ; a+}^{\gamma} \phi\right)(\tau)\right\| \leq Q\|\phi\|,
$$

where

$$
Q=(b-a)^{\operatorname{Re}(\mu)} \sum_{k=0}^{\infty} \frac{\left|(\gamma)_{k}\right|}{|\Gamma(\rho k+\mu)|[(\operatorname{Re}(\rho) k+\operatorname{Re}(\mu)]} \frac{\left|\omega(b-a)^{\operatorname{Re}(\rho)}\right|^{k}}{k !} .
$$

Lemma $2.4[46,47]$ Let $\rho, \mu, \omega, \gamma \in \mathbb{C}(\operatorname{Re}(\rho), \operatorname{Re}(\mu)>0)$, then the operator $\mathcal{E}_{\rho, \mu, \omega ; a+}^{\gamma}$ is invertible in the space $L(a, b)$ and for $f \in L(a, b)$ its left inversion is given by the relation

$$
\left(\left[\mathcal{E}_{\rho, \mu, \omega ; a+}^{\gamma}\right]^{-1} f\right) \tau=\left(\mathcal{D}_{a+}^{\mu+v} \mathcal{E}_{\rho, \nu, \omega ; a+}^{-\gamma} f\right)(\tau), a<\tau \leq b,
$$

where $v \in \mathbb{C},(\operatorname{Re}(v)>0)$ and $\mathcal{D}_{a+}^{\mu+v}$ is the Riemann-Liouville fractional differential operator of order $\mu+v$ with lower terminal a.

Lemma $2.5[46,47]$ Let $\rho, \mu, \omega, \gamma \in \mathbb{C}(\operatorname{Re}(\rho), \operatorname{Re}(\mu)>0)$. If the integral equation

$$
\int_{a}^{\tau}(\tau-\sigma)^{\gamma-1} \mathbb{E}_{\rho, \mu}^{\gamma}\left[\omega(\tau-\sigma)^{\alpha}\right] \phi(\sigma) d \sigma=f(\tau), a<\tau \leq b,
$$

is solvable in the space $L(a, b)$, then its unique solution $\phi(\tau)$ is given by

$$
\phi(\tau)=\left(\mathcal{D}_{a+}^{\mu+v} \mathcal{E}_{\rho, v, \omega ; a+}^{-\gamma} f\right)(\tau), a<\tau \leq b,
$$

where $v \in \mathbb{C},(\operatorname{Re}(v)>0)$ and $\mathcal{D}_{a+}^{\mu+v}$ is the Riemann-Liouville fractional differential operator of order $\mu+v$ with lower terminal a.

Lemma 2.6 (Krasnoselskii's Fixed Point Theorem [15]) Let $\Omega$ be a Banach space. Let $\mathcal{S}$ be a bounded, closed, convex subset of $\Omega$ and let $\mathcal{F}_{1}, \mathcal{F}_{2}$ be maps of $\mathcal{S}$ into $\Omega$ such that $\mathcal{F}_{1} \omega+\mathcal{F}_{2} \eta \in \mathcal{S}$ for every pair $\omega, \eta \in \mathcal{S}$. If $\mathcal{F}_{1}$ is contraction and $\mathcal{F}_{2}$ is completely continuous, then the equation

$$
\mathcal{F}_{1} \omega+\mathcal{F}_{2} \omega=\omega
$$

has a solution on $\mathcal{S}$. 
Lemma 2.7 ( Gronwall-Bellman inequality [48]) Let $u$ and $f$ be continuous and nonnegative functions defined on $J=[\alpha, \beta]$, and let $c$ be a nonnegative constant. Then the inequality

$$
u(\tau) \leq C+\int_{\alpha}^{\tau} f(\sigma) u(\sigma) d \sigma,, \tau \in J
$$

implies that

$$
u(\tau) \leq C \exp \left(\int_{\alpha}^{\tau} f(\sigma) d \sigma\right), \tau \in J
$$

\section{Equivalent fractional integral equation}

In this section we obtain an equivalent fractional integral equation to the ABR-FDEs (1.1)(1.2) by analytical method and then by using by the method of Laplace transform.

Theorem 3.1 For any function $h \in C(J)$, the function $\omega \in C(J)$ is a solution of ABR-FDEs

$$
\begin{gathered}
\frac{d \omega}{d \tau}+{ }_{0}^{*} \mathcal{D}_{\tau}^{\alpha} \omega(\tau)=h(\tau), \tau \in J, \\
\omega(0)=\omega_{0} \in \mathbb{R},
\end{gathered}
$$

if and only if $\omega$ is a solution of fractional integral equation

$$
\omega(\tau)=\omega_{0}-\frac{B(\alpha)}{1-\alpha} \int_{0}^{\tau} \mathbb{E}_{\alpha}\left[\frac{-\alpha}{1-\alpha}(\tau-\sigma)^{\alpha}\right] \omega(\sigma) d \sigma+\int_{0}^{\tau} h(\sigma) d \sigma, \tau \in J .
$$

Proof 1:

Using definition of ABR-fractional derivative, Eq.(3.1) can be written as

$$
\frac{d}{d \tau}\left(\omega(\tau)+\frac{B(\alpha)}{1-\alpha} \int_{0}^{\tau} \mathbb{E}_{\alpha}\left[-\frac{\alpha}{1-\alpha}(\tau-\sigma)^{\alpha}\right] \omega(\sigma) d \sigma\right)=h(\tau), \tau \in J
$$

Integrating both sides of above equation between the limits 0 to $\tau$, we obtain

$$
\omega(\tau)+\frac{B(\alpha)}{1-\alpha} \int_{0}^{\tau} \mathbb{E}_{\alpha}\left[-\frac{\alpha}{1-\alpha}(\tau-\sigma)^{\alpha}\right] \omega(\sigma) d \sigma-\omega(0)=\int_{0}^{\tau} h(\sigma) d \sigma, \tau \in J,
$$

which gives desired fractional integral Eq.(3.3).

Conversely, if $\omega \in C(J)$ satisfies fractional integral Eq.(3.3), then differentiating both sides of Eq.(3.3) with respect to $\tau$, we obtain

$$
\frac{d \omega}{d \tau}+\frac{B(\alpha)}{1-\alpha} \frac{d}{d \tau} \int_{0}^{\tau} \mathbb{E}_{\alpha}\left[-\frac{\alpha}{1-\alpha}(\tau-\sigma)^{\alpha}\right] \omega(\sigma) d \sigma=h(\tau), \tau \in J .
$$

Using definition of ABR-fractional derivative, we get Eq. (3.1). Further putting $\tau=0$ in Eq. (3.3), we get initial condition (3.2).

Proof 2: Taking Laplace transform of both sides of Eq.(3.1), we get 


$$
\mathcal{L}\left[\omega^{\prime}(\tau) ; p\right]+\mathcal{L}\left[{ }_{0}^{*} \mathcal{D}_{\tau}^{\alpha} \omega(\tau) ; p\right]=\mathcal{L}[h(\tau) ; p] .
$$

Then using formula for Laplace transform of ordinary and ABR-fractional derivative given in Lemma 2.1, we get

$$
p \bar{W}(p)-\omega(0)+\frac{B(\alpha)}{1-\alpha} \frac{p^{\alpha} \bar{W}(p)}{p^{\alpha}+\frac{\alpha}{1-\alpha}}=\bar{H}(p),
$$

where $\bar{W}(p)=\mathcal{L}[\omega(\tau) ; p]$ and $\bar{H}(p)=\mathcal{L}[h(\tau) ; p]$. Using initial condition (3.2), we rewrite the above equation as

$$
\bar{W}(p)=\omega_{0} \frac{1}{p}-\frac{B(\alpha)}{1-\alpha} \frac{p^{\alpha-1} \bar{W}(p)}{p^{\alpha}+\frac{\alpha}{1-\alpha}}+\bar{H}(p) \frac{1}{p} .
$$

Now taking inverse Laplace transform on both sides of above equation and using convolution theorem, Lemma 2.2, we obtain

$$
\begin{aligned}
\mathcal{L}^{-1}[\bar{W}(p) ; \tau]= & \omega_{0} \mathcal{L}^{-1}\left[\frac{1}{p} ; \tau\right]-\frac{B(\alpha)}{1-\alpha}\left(\mathcal{L}^{-1}\left[\frac{p^{\alpha-1}}{p^{\alpha}+\frac{\alpha}{1-\alpha}} ; \tau\right] * \mathcal{L}^{-1}[\bar{W}(p) ; \tau]\right) \\
& +\mathcal{L}^{-1}[\bar{H}(p) ; \tau] * \mathcal{L}^{-1}\left[\frac{1}{p} ; \tau\right] \\
= & \omega_{0}-\frac{B(\alpha)}{1-\alpha}\left(\mathbb{E}_{\alpha}\left[\frac{-\alpha}{1-\alpha} \tau^{\alpha}\right] * \omega(\tau)\right)+h(\tau) * 1 \\
= & \omega_{0}-\frac{B(\alpha)}{1-\alpha} \int_{0}^{\tau} \mathbb{E}_{\alpha}\left[\frac{-\alpha}{1-\alpha}(\tau-\sigma)^{\alpha}\right] \omega(\sigma) d \sigma+\int_{0}^{\tau} h(\sigma) d \sigma .
\end{aligned}
$$

From above equation, we have

$$
\omega(\tau)=\omega_{0}-\frac{B(\alpha)}{1-\alpha} \int_{0}^{\tau} \mathbb{E}_{\alpha}\left[\frac{-\alpha}{1-\alpha}(\tau-\sigma)^{\alpha}\right] \omega(\sigma) d \sigma+\int_{0}^{\tau} h(\sigma) d \sigma .
$$

which is desired fractional integral Eq. (3.3).

Remark 3.2 Using the definition of fractional integral operator $\mathcal{E}_{\rho, \mu, \omega ; a+}^{\gamma}$, the equivalent fractional integral equation (3.4) to the ABR-FDEs (3.1)-(3.2) is given by

$$
\omega(\tau)=\omega_{0}-\frac{B(\alpha)}{1-\alpha}\left(\mathcal{E}_{\alpha, 1, \frac{\alpha}{1-\alpha} ; 0+}^{1} \omega\right)(\tau)+\int_{0}^{\tau} h(\sigma) d \sigma, \tau \in J .
$$

Theorem 3.3 For any $f \in C(J \times \mathbb{R}, \mathbb{R})$, the function $\omega \in C(J)$ is a solution of ABR-FDEs (1.1)-(1.2) if and only if $\omega$ is a solution of fractional integral equation

$$
\omega(\tau)=\omega_{0}-\frac{B(\alpha)}{1-\alpha} \int_{0}^{\tau} \mathbb{E}_{\alpha}\left[\frac{-\alpha}{1-\alpha}(\tau-\sigma)^{\alpha}\right] \omega(\sigma) d \sigma+\int_{0}^{\tau} f(\sigma, \omega(\sigma)) d \sigma, \tau \in J .
$$

Proof Proof follows by taking $h(\tau)=f(\tau, \omega(\tau)), \tau \in J$, in the Theorem 3.1.

The proof of following theorem is based on the properties of fractional integral operator $\mathcal{E}_{\rho, \mu, \omega ; a+}^{\gamma}$ studied in $[46,47]$. 


\section{Existence and uniqueness results}

Theorem 4.1 Let $0<\alpha<1$. Define the function $\mathcal{F}$ on $C(J)$ by

$$
(\mathcal{F} \omega)(\tau)=\frac{B(\alpha)}{1-\alpha}\left(\mathcal{E}_{\alpha, 1, \frac{-\alpha}{1-\alpha} ; 0+}^{1} \omega\right)(\tau), \omega \in C(J), \tau \in J .
$$

Then:

(i) $\mathcal{F}$ is bounded linear operator on $C(J)$.

(ii) $\mathcal{F}$ satisfies Lipschitz condition.

(iii) $\mathcal{F}(\mathcal{S})$ is equicontinuous, where $\mathcal{S}$ is any bounded subset of $C(J)$.

(iv) $\mathcal{F}$ is invertible and for any $f \in C(J)$, the operator equation $\mathcal{F} \omega=f$ has unique solution in $C(J)$.

Proof (i) Since, by definition and Lemma 2.3, the integral operator $\mathcal{E}_{\alpha, 1, \frac{-\alpha}{1-\alpha} ; 0+}^{1}$ is bounded and linear operator on $C(J)$, such that

$$
\left\|\mathcal{E}_{\alpha, 1, \frac{-\alpha}{1-\alpha} ; 0+}^{1} \omega\right\| \leq Q\|\omega\|, \tau \in J
$$

where we find

$$
Q=T \sum_{k=0}^{\infty} \frac{(1)_{k}}{\Gamma(\alpha k+1)(\alpha k+1)} \frac{\left|\frac{-\alpha}{1-\alpha} T^{\alpha}\right|^{k}}{k !}=T \sum_{k=0}^{\infty} \frac{\left(\frac{\alpha}{1-\alpha}\right)^{k} T^{\alpha^{k}}}{\Gamma(\alpha k+2)}=T \mathbb{E}_{\alpha, 2}\left(\frac{\alpha}{1-\alpha} T^{\alpha}\right),
$$

we have

$$
\|\mathcal{F} \omega\|=\left|\frac{B(\alpha)}{1-\alpha}\right|\left\|\mathcal{E}_{\alpha, 1, \frac{-\alpha}{1-\alpha} ; 0+}^{1} \omega\right\| \leq Q \frac{B(\alpha)}{1-\alpha}\|\omega\|, \text { for all } \omega \in C(J)
$$

Thus $\mathcal{F}$ is bounded linear operator on $C(J)$.

(ii) Let any $\omega, \eta \in C(J)$. Then using linearity of $\mathcal{F}$ and boundedness of operator $\mathcal{E}_{\alpha, 1, \frac{-\alpha}{1-\alpha} ; 0+}^{1}$, we find for any $\tau \in J$,

$$
\begin{aligned}
|(\mathcal{F} \omega)(\tau)-(\mathcal{F} \eta)(\tau)| & =|(\mathcal{F}(\omega-\eta))(\tau)|=\frac{B(\alpha)}{1-\alpha}\left|\left(\mathcal{E}_{\alpha, 1, \frac{-\alpha}{1-\alpha} ; 0+}^{1} \omega-\eta\right)(\tau)\right| \\
& \leq \frac{B(\alpha)}{1-\alpha}\left\|\mathcal{E}_{\alpha, 1, \frac{-\alpha}{1-\alpha} ; 0+}^{1}(\omega-\eta)\right\| \leq Q \frac{B(\alpha)}{1-\alpha}\|\omega-\eta\| .
\end{aligned}
$$

This gives

$$
\|\mathcal{F} \omega-\mathcal{F} \eta\| \leq Q \frac{B(\alpha)}{1-\alpha}\|\omega-\eta\|, \omega, \eta \in C(J)
$$

Thus the operator $\mathcal{F}$ satisfies Lipschitz condition with Lipschitz constant $T \frac{B(\alpha)}{1-\alpha} \mathbb{E}_{\alpha, 2}\left(\frac{\alpha}{1-\alpha} T^{\alpha}\right)$.

(iii) Let $\mathcal{S}=\{\omega \in C(J):\|\omega\| \leq R\}$ be any closed, bounded subset of $C(J)$. Then for any $\omega \in \mathcal{S}$ and any $\tau_{1}, \tau_{2} \in J$ with $\tau_{1}<\tau_{2}$, we find 


$$
\begin{aligned}
& \left|(\mathcal{F} \omega) \tau_{1}-(\mathcal{F} \omega) \tau_{2}\right|=\left|\frac{B(\alpha)}{1-\alpha}\left(\mathcal{E}_{\alpha, 1, \frac{-\alpha}{1-\alpha} ; 0+}^{1} \omega\right)\left(\tau_{1}\right)-\frac{B(\alpha)}{1-\alpha}\left(\mathcal{E}_{\alpha, 1, \frac{-\alpha}{1-\alpha} ; 0+}^{1} \omega\right)\left(\tau_{2}\right)\right| \\
& =\frac{B(\alpha)}{1-\alpha}\left|\int_{0}^{\tau_{1}} \mathbb{E}_{\alpha}\left[-\frac{\alpha}{1-\alpha}\left(\tau_{1}-\sigma\right)^{\alpha}\right] \omega(\sigma) d \sigma-\int_{0}^{\tau_{2}} \mathbb{E}_{\alpha}\left[-\frac{\alpha}{1-\alpha}\left(\tau_{2}-\sigma\right)^{\alpha}\right] \omega(\sigma) d \sigma\right| \\
& \leq \frac{B(\alpha)}{1-\alpha}\left|\int_{0}^{\tau_{1}}\left\{\mathbb{E}_{\alpha}\left[-\frac{\alpha}{1-\alpha}\left(\tau_{1}-\sigma\right)^{\alpha}\right]-\mathbb{E}_{\alpha}\left[-\frac{\alpha}{1-\alpha}\left(\tau_{2}-\sigma\right)^{\alpha}\right]\right\} \omega(\sigma) d \sigma\right| \\
& \left.\quad+\frac{B(\alpha)}{1-\alpha} \mid \int_{\tau_{1}}^{\tau_{2}} \mathbb{E}_{\alpha}\left[-\frac{\alpha}{1-\alpha}\left(\tau_{2}-\sigma\right)^{\alpha}\right] \omega(\sigma) d \sigma\right) \\
& \leq \frac{B(\alpha)}{1-\alpha} \sum_{k=0}^{\infty}\left|\left(\frac{-\alpha}{1-\alpha}\right)^{k}\right| \frac{1}{\Gamma(k \alpha+1)} \int_{0}^{\tau_{1}}\left|\left(\tau_{1}-\sigma\right)^{k \alpha}-\left(\tau_{2}-\sigma\right)^{k \alpha}\right||\omega(\sigma)| d \sigma \\
& \quad+\frac{B(\alpha)}{1-\alpha} \sum_{k=0}^{\infty}\left|\left(\frac{-\alpha}{1-\alpha}\right)^{k}\right| \frac{1}{\Gamma(k \alpha+1)} \int_{\tau_{1}}^{\tau_{2}}\left|\left(\tau_{2}-\sigma\right)^{k \alpha}\right||\omega(\sigma)| d \sigma \\
& \leq \frac{R B(\alpha)}{1-\alpha} \sum_{k=0}^{\infty}\left(\frac{\alpha}{1-\alpha}\right)^{k} \frac{1}{\Gamma(k \alpha+1)} \int_{0}^{\tau_{1}}\left\{\left(\tau_{2}-\sigma\right)^{k \alpha}-\left(\tau_{1}-\sigma\right)^{k \alpha}\right\} d \sigma \\
& \quad+\frac{R B(\alpha)}{1-\alpha} \sum_{k=0}^{\infty}\left(\frac{\alpha}{1-\alpha}\right)^{k} \frac{1}{\Gamma(k \alpha+1)} \int_{\tau_{1}}^{\tau_{2}}\left(\tau_{2}-\sigma\right)^{k \alpha} d \sigma \\
& \leq \frac{R B(\alpha)}{1-\alpha} \sum_{k=0}^{\infty}\left(\frac{\alpha}{1-\alpha}\right)^{k} \frac{1}{\Gamma(k \alpha+2)}\left\{-\left(\tau_{2}-\tau_{1}\right)^{k \alpha+1}+\tau_{2}^{k \alpha+1}-\tau_{1}^{k \alpha+1}+\left(\tau_{2}-\tau_{1}\right)^{k \alpha+1}\right\} \\
& \leq \frac{R B(\alpha)}{1-\alpha} \sum_{k=0}^{\infty}\left(\frac{\alpha}{1-\alpha}\right)^{k} \frac{1}{\Gamma(k \alpha+2)}\left\{\tau_{2}^{k \alpha+1}-\tau_{1}^{k \alpha+1}\right\} .
\end{aligned}
$$

From above inequality it follows that, if $\left|\tau_{1}-\tau_{2}\right| \rightarrow 0$ then $\left|(\mathcal{F} \omega) \tau_{1}-(\mathcal{F} \omega) \tau_{2}\right| \rightarrow 0$. This prove that $\mathcal{F}(\mathcal{S})$ is equicontinious on $J$.

(iv) Using Lemma 2.4 and Lemma 2.5, for any $f \in C(J)$, we have

$$
\left(\mathcal{E}_{\alpha, 1, \frac{-\alpha}{1-\alpha} ; 0+}^{1} f\right)^{-1}(\tau)=\left(\mathcal{D}_{0+}^{1+\beta} \mathcal{E}_{\alpha, \beta, \frac{-\alpha}{1-\alpha} ; 0+}^{-1} f\right)(\tau), \tau \in(a, b)
$$

where $\beta \in \mathbb{C}$, with $\operatorname{Re}(\beta)>0$.

Then using definition of operator $\mathcal{F}$ and Eq. (4.2), we have

$$
\left(\mathcal{F}^{-1} f\right)(\tau)=\left(\frac{B(\alpha)}{1-\alpha} \mathcal{E}_{\alpha, 1, \frac{-\alpha}{1-\alpha} ; 0+}^{1} f\right)^{-1}(\tau)=\frac{1-\alpha}{B(\alpha)}\left(\mathcal{D}_{0+}^{1+\beta} \mathcal{E}_{\alpha, \beta, \frac{-\alpha}{1-\alpha} ; 0+}^{-1} f\right)(\tau), \tau \in(a, b) .
$$

This prove that $\mathcal{F}$ is invertible on $C(J)$ and the operator equation

$$
(\mathcal{F} \omega)(\tau)=f(\tau), \tau \in J,
$$

has the unique solution

$$
\omega(\tau)=\left(\mathcal{F}^{-1} f\right)(\tau)=\frac{1-\alpha}{B(\alpha)}\left(\mathcal{D}_{0+}^{1+\beta} \mathcal{E}_{\alpha, \beta, \frac{-\alpha}{1-\alpha} ; 0+}^{-1} f\right)(\tau), \tau \in(a, b)
$$

We have the following existence theorem for the particular case of ABR-FDEs (1.1) 
Theorem 4.2 If the function $f \in C(J \times \mathbb{R}, \mathbb{R})$, then ABR-FDEs ${ }_{0}^{*} \mathcal{D}_{\tau}^{\alpha} \omega(\tau)=f(\tau, \omega(\tau))$, $\tau \in J$ is solvable in $C(J)$ and has solution in $C(J)$ given by

$$
\omega(\tau)=\frac{1-\alpha}{B(\alpha)}\left(\mathcal{D}_{0+}^{1+\beta} \mathcal{E}_{\alpha, \beta, \frac{-\alpha}{1-\alpha} ; 0+}^{-1} \tilde{f}\right)(\tau), \tau \in J,
$$

where $\beta \in \mathbb{C},(\operatorname{Re}(\beta)>0)$ and $\tilde{f}(\tau)=\int_{0}^{\tau} f(\sigma, \omega(\sigma)) d \sigma, \tau \in J$.

Proof The equivalent integral equation of ABR-FDE

$$
{ }_{0}^{*} \mathcal{D}_{\tau}^{\alpha} \omega(\tau)=f(\tau, \omega(\tau)), \tau \in J,
$$

is given by

$$
\frac{B(\alpha)}{1-\alpha} \int_{0}^{\tau} \mathbb{E}_{\alpha}\left[-\frac{\alpha}{1-\alpha}(\tau-\sigma)^{\alpha}\right] \omega(\sigma) d \sigma=\int_{0}^{\tau} f(\sigma, \omega(\sigma)) d \sigma, \tau \in J .
$$

Using definition of operator $\mathcal{F}$ defined in Eq. (4.1), above equation can be written as

$$
(\mathcal{F} \omega)(\tau)=\int_{0}^{\tau} f(\sigma, \omega(\sigma)) d \sigma=\tilde{f}(\tau), \tau \in J .
$$

By Theorem 4.1, the operator Eq. (4.3) is solvable and has a solution in $C(J)$ given by

$$
\omega(\tau)=\frac{1-\alpha}{B(\alpha)}\left(\mathcal{D}_{0+}^{1+\beta} \mathcal{E}_{\alpha, \beta, \frac{-\alpha}{1-\alpha} ; 0+}^{-1} \tilde{f}\right)(\tau), \tau \in J ; \beta \in \mathbb{C}, \operatorname{Re}(\beta)>0 .
$$

Now we derive existence and uniqueness results to the ABR-FDEs (1.1)-(1.2).

Theorem 4.3 (Existence Theorem.) Let the function $f \in C(J \times \mathbb{R}, \mathbb{R})$ satisfies Lipschitz type condition

$$
|f(\tau, \omega)-f(\tau, \eta)| \leq p(\tau)|\omega-\eta|, \omega, \eta \in C(J),
$$

where $p: J \rightarrow \mathbb{R}^{+}$, with $L=\sup _{\tau \in J} p(\tau)$. If $0<L<\min \left\{1, \frac{1}{2 T}\right\}$, then ABR-FDEs (1.1)(1.2) has a solution in $C(J)$ provided

$$
\frac{2 B(\alpha) T \mathbb{E}_{\alpha, 2}\left(\frac{\alpha}{1-\alpha} T^{\alpha}\right)}{1-\alpha}<1 .
$$

Proof Define,

$$
R=\frac{\left|\omega_{0}\right|+M_{f} T}{1-L T-\frac{B(\alpha) T \mathbb{E}_{\alpha, 2}\left(\frac{\alpha}{1-\alpha} T^{\alpha}\right)}{1-\alpha}}, \text { where } M_{f}=\sup _{\tau \in J}|f(\tau, 0)| .
$$

By the choice of $L$ and condition (4.4), we have $R>0$.

Consider the set,

$$
\mathcal{S}=\{\omega \in C(J):\|\omega\| \leq R\}
$$


One can verify that $\mathcal{S}$ is closed, convex and bounded subset of Banach space $\Omega$. Consider the operators $\mathcal{F}_{1}: \mathcal{S} \rightarrow \Omega$ and $\mathcal{F}_{2}: \mathcal{S} \rightarrow \Omega$ defined by,

$$
\begin{aligned}
& \left(\mathcal{F}_{1} \omega\right)(\tau)=\omega_{0}+\int_{0}^{\tau} f(\sigma, \omega(\sigma)) d \sigma, \tau \in J \\
& \left(\mathcal{F}_{2} \omega\right)(\tau)=-(\mathcal{F} \omega)(\tau), \tau \in J
\end{aligned}
$$

where we take $\mathcal{F}$ as defined in the Eq.(4.1). The equivalent fractional integral Eq.(3.4) to the ABR-FDEs (1.1)-(1.2) can be written as operator equation in the following form

$$
\omega=\mathcal{F}_{1} \omega+\mathcal{F}_{2} \omega, \omega \in C(J) .
$$

We prove that the operators $\mathcal{F}_{1}$ and $\mathcal{F}_{2}$ satisfies conditions of Lemma 2.6. The proof of the same have been given in following steps.

Step 1) $\mathcal{F}_{1}$ is contraction.

Using Lipschitz condition on $f$, for any $\omega, \eta \in C(J)$ and $\tau \in J$ we obtain,

$$
|\mathcal{F}(\tau, \omega(\tau))-\mathcal{F}(\tau, \eta(\tau))| \leq p(\tau)|\omega(\tau)-n(\tau)| \leq L|| \omega-\eta \|,
$$

This gives,

$$
\left\|\mathcal{F}_{1} \omega-\mathcal{F}_{1} \eta\right\| \leq L T\|\omega-\eta\|, \omega, \eta \in C(J) .
$$

Step 2) $\mathcal{F}_{2}$ is completely continuous.

Using Ascoli-Arzela theorem and Theorem 4.1, one can easily verify that the operator $\mathcal{F}_{2}=-\mathcal{F}$ is completely continuous.

Step 3) $\mathcal{F}_{1} \omega+\mathcal{F}_{2} \eta \in \mathcal{S}$, for $\omega, \eta \in \mathcal{S}$.

For any $\omega, \eta \in \mathcal{S}$, using Theorem 4.1, we obtain

$$
\begin{aligned}
& \left|\left(\mathcal{F}_{1} \omega+\mathcal{F}_{2} \eta\right)(\tau)\right| \leq\left|\left(\mathcal{F}_{1} \omega\right)(\tau)\right|+\left|\left(\mathcal{F}_{2} \eta\right)(\tau)\right| \\
& \leq\left|\omega_{0}\right|+\int_{0}^{\tau}|f(\sigma, \omega(\sigma))| d \sigma+\frac{B(\alpha)}{1-\alpha} T \mathbb{E}_{\alpha, 2}\left(\frac{\alpha}{1-\alpha} T^{\alpha}\right)\|\eta\| \\
& \leq\left|\omega_{0}\right|+\int_{0}^{\tau}|f(\sigma, \omega(\sigma))-f(\sigma, 0)| d \sigma+\int_{0}^{\tau}|f(\sigma, 0)| d \sigma+\frac{B(\alpha)}{1-\alpha} T \mathbb{E}_{\alpha, 2}\left(\frac{\alpha}{1-\alpha} T^{\alpha}\right) R \\
& \left.\leq\left|\omega_{0}\right|+L \int_{0}^{\tau} \mid \omega(\sigma)\right) \mid d \sigma+M_{f} \int_{0}^{\tau} d \sigma+\frac{B(\alpha)}{1-\alpha} T \mathbb{E}_{\alpha, 2}\left(\frac{\alpha}{1-\alpha} T^{\alpha}\right) R \\
& \leq\left|\omega_{0}\right|+L R \tau+M_{f} \tau+\frac{B(\alpha)}{1-\alpha} T \mathbb{E}_{\alpha, 2}\left(\frac{\alpha}{1-\alpha} T^{\alpha}\right) R \\
& \leq\left|\omega_{0}\right|+L R T+M_{f} T+\frac{B(\alpha)}{1-\alpha} T \mathbb{E}_{\alpha, 2}\left(\frac{\alpha}{1-\alpha} T^{\alpha}\right) R .
\end{aligned}
$$

By definition of $R$ i.e. condition(4.4), we get

$$
\left|\omega_{0}\right|+M_{f} T=R\left(1-L T-\frac{B(\alpha) T \mathbb{E}_{\alpha, 2}\left(\frac{\alpha}{1-\alpha} T^{\alpha}\right)}{1-\alpha}\right) .
$$

We write from inequalities (4.5) and (4.6)

$$
\left|\left(\mathcal{F}_{1} \omega+\mathcal{F}_{2} \eta\right)(\tau)\right| \leq R, \tau \in J .
$$

This gives 


$$
\left\|\mathcal{F}_{1} \omega+\mathcal{F}_{2} \eta\right\| \leq R, \text { for all } \omega, \eta \in \mathcal{S} .
$$

This shows that $\mathcal{F}_{1} \omega+\mathcal{F}_{2} \eta \in \mathcal{S}$, for $\omega, \eta \in \mathcal{S}$.

From steps 1 to 3 , it follows that all the conditions of Lemma 2.6 are satisfied. Therefore by applying it, the operator equation

$$
\omega=\mathcal{F}_{1} \omega+\mathcal{F}_{2} \omega,
$$

has a fixed point in $\mathcal{S}$, which is a solution of ABR-FDEs (1.1)-(1.2). This completes the proof of the theorem.

In the following theorem we prove the uniqueness of solution to ABR-FDEs (1.1)-(1.2) in two different ways. Firstly we give the proof via properties of fractional integral operator $\mathcal{E}_{\alpha, 1, \frac{-\alpha}{1-\alpha} ; 0+}^{1}$ and then by using the Gronwall-Bellman inequality.

Theorem 4.4 (Uniqueness Result) Under the assumptions of Theorem 4.3, the ABR-FDEs (1.1)-(1.2) has unique solution in $C(J)$.

\section{Proof 1:}

The equivalent fractional integral equation to ABR-FDEs (1.1)-(1.2) can be written in operator equation form as

$$
\left(\mathcal{E}_{\alpha, 1, \frac{-\alpha}{1-\alpha} ; 0+}^{1} \omega\right)(\tau)=\tilde{f}(\tau), \tau \in J
$$

where

$$
\tilde{f}(\tau)=\frac{1-\alpha}{B(\alpha)}\left(\omega_{0}-\omega(\tau)+\int_{0}^{\tau} f(\sigma, \omega(\sigma)) d \sigma\right), \tau \in J
$$

By Theorem 4.3, the operator Eq.(4.7) is solvable in $C(J)$. Therefore by applying Lemma 2.5, the operator equation Eq.(4.7) has unique solution in $C(J)$, which is the unique solution of ABR-FDEs (1.1)-(1.2).

\section{Proof 2:}

Let $\omega, \eta$ be two solutions of ABR-FDEs (1.1)-(1.2). Using linearity of fractional integral operator, we find for any $\tau \in J$

$$
\begin{aligned}
|\omega(\tau)-\eta(\tau)|= & \mid\left(\omega_{0}-\frac{B(\alpha)}{1-\alpha}\left(\mathcal{E}_{\alpha, 1, \frac{-\alpha}{1-\alpha} ; 0+}^{1} \omega\right)(\tau)+\int_{0}^{\tau} f(\sigma, \omega(\sigma)) d \sigma\right) \\
& -\left(\omega_{0}-\frac{B(\alpha)}{1-\alpha}\left(\mathcal{E}_{\alpha, 1, \frac{-\alpha}{1-\alpha} ; 0+}^{1} \eta\right)(\tau)+\int_{0}^{\tau} f(\sigma, \eta(\sigma)) d \sigma\right) \mid \\
\leq & \left|\frac{B(\alpha)}{1-\alpha}\left(\mathcal{E}_{\alpha, 1, \frac{-\alpha}{1-\alpha} ; 0+}^{1}(\omega-\eta)\right)(\tau)\right|+\int_{0}^{\tau}|f(\sigma, \omega(\sigma))-f(\sigma, \eta(\sigma))| d \sigma \\
\leq & \frac{B(\alpha)}{1-\alpha} \int_{0}^{\tau} \mathbb{E}_{\alpha}\left(\left|\frac{-\alpha}{1-\alpha}(\tau-\sigma)^{\alpha}\right|\right)|\omega(\sigma)-\eta(\sigma)| d \sigma+\int_{0}^{\tau} p(\sigma)|\omega(\sigma)-\eta(\sigma)| d \sigma \\
\leq & \frac{B(\alpha)}{1-\alpha} \int_{0}^{\tau} \mathbb{E}_{\alpha}\left(\frac{\alpha}{1-\alpha} T^{\alpha}\right)|\omega(\sigma)-\eta(\sigma)| d \sigma+\int_{0}^{\tau} p(\sigma)|\omega(\sigma)-\eta(\sigma)| d \sigma \\
\leq & \int_{0}^{\tau}\left[\frac{B(\alpha)}{1-\alpha} \mathbb{E}_{\alpha}\left(\frac{\alpha}{1-\alpha} T^{\alpha}\right)+p(\sigma)\right]|\omega(\sigma)-\eta(\sigma)| d \sigma .
\end{aligned}
$$


Applying Lemma 2.7, we get

$$
|\omega(\tau)-\eta(\tau)| \leq 0, \tau \in J
$$

which shows that $\omega(\tau)=\eta(\tau)$, for all $\tau \in J$. This proves the uniqueness of solution of ABR-FDEs (1.1)-(1.2).

\section{Estimate on solution and data dependence}

Theorem 5.1 Under the assumptions of Theorem 4.3, if $\omega(\tau)$ is a solution of ABR-FDEs (1.1)-(1.2), then

$$
|\omega(\tau)| \leq\left\{\left|\omega_{0}\right|+M_{f} T\right\} \exp \left(\int_{0}^{\tau}\left[\frac{B(\alpha)}{1-\alpha} \mathbb{E}_{\alpha}\left(\frac{\alpha}{1-\alpha} T^{\alpha}\right)+p(\sigma)\right] d \sigma\right), \tau \in J .
$$

Proof If $\omega(\tau)$ is a solution of ABR-FDEs (1.1)-(1.2), then it satisfies equivalent intgeral Eq.(3.4). Hence we write for any $\tau \in J$,

$$
\begin{aligned}
|\omega(\tau)| \leq & \left|\omega_{0}\right|+\frac{B(\alpha)}{1-\alpha} \int_{0}^{\tau} \mathbb{E}_{\alpha}\left(\left|\frac{-\alpha}{1-\alpha}(\tau-\sigma)^{\alpha}\right|\right)|\omega(\sigma)| d \sigma+\int_{0}^{\tau}|f(\sigma, \omega(\sigma))| d \sigma \\
\leq & \left|\omega_{0}\right|+\frac{B(\alpha)}{1-\alpha} \int_{0}^{\tau} \mathbb{E}_{\alpha}\left(\frac{\alpha}{1-\alpha}(\tau-\sigma)^{\alpha}\right)|\omega(\sigma)| d \sigma+\int_{0}^{\tau}|f(\sigma, \omega(\sigma))-f(\sigma, 0)| d \sigma \\
& \quad+\int_{0}^{\tau}|f(\sigma, 0)| d \sigma \\
\leq & \left|\omega_{0}\right|+\frac{B(\alpha)}{1-\alpha} \int_{0}^{\tau} \mathbb{E}_{\alpha}\left(\frac{\alpha}{1-\alpha} T^{\alpha}\right)|\omega(\sigma)| d \sigma+\int_{0}^{\tau} p(\sigma)|\omega(\sigma)| d \sigma+M_{f} \tau \\
= & \left\{\left|\omega_{0}\right|+M_{f} T\right\}+\int_{0}^{\tau}\left[\frac{B(\alpha)}{1-\alpha} \mathbb{E}_{\alpha}\left(\frac{\alpha}{1-\alpha} T^{\alpha}\right)+p(\sigma)\right]|\omega(\sigma)| d \sigma
\end{aligned}
$$

Applying Lemma 2.7, we obtain

$$
|\omega(\tau)| \leq\left\{\left|\omega_{0}\right|+M_{f} T\right\} \exp \left(\int_{0}^{\tau}\left[\frac{B(\alpha)}{1-\alpha} \mathbb{E}_{\alpha}\left(\frac{\alpha}{1-\alpha} T^{\alpha}\right)+p(\sigma)\right] d \sigma\right), \tau \in J .
$$

In order to discuss the data dependence result, we consider ABR-FDEs

$$
\begin{gathered}
\frac{d \eta}{d \tau}+{ }_{0}^{*} \mathcal{D}_{\tau}^{\alpha} \eta(\tau)=\bar{f}(\tau, \eta(\tau)), \tau \in J, \\
\eta(0)=\eta_{0} \in \mathbb{R} .
\end{gathered}
$$


Theorem 5.2 Assume the conditions of Theorem 4.3 holds. Let $\epsilon_{i}>0, i=1,2$ be any two real numbers such that,

$$
\left|\omega_{0}-\eta_{0}\right| \leq \epsilon_{1},|f(\tau, \eta(\tau))-\bar{f}(\tau, \eta(\tau))| \leq \epsilon_{2}, \tau \in J,
$$

where $\eta(\tau)$ is a solution of ABR-FDEs (5.2)-(5.3). Then, the solution $\omega(\tau)$ of (1.1)-(1.2) depends continuously on the function involved on the right side of Eq. (1.1).

Proof Since $\omega, \eta$ are the solutions of ABR-FDEs (1.1)-(1.2) and (5.2)-(5.3) receptively. We find for any $\tau \in J$

$$
\begin{aligned}
& |\omega(\tau)-\eta(\tau)|=\mid\left(\omega_{0}-\frac{B(\alpha)}{1-\alpha} \int_{0}^{\tau} \mathbb{E}_{\alpha}\left(\frac{-\alpha}{1-\alpha}(\tau-\sigma)^{\alpha}\right) \omega(\sigma) d \sigma+\int_{0}^{\tau} f(\sigma, \omega(\sigma)) d \sigma\right) \\
& -\left(\eta_{0}-\frac{B(\alpha)}{1-\alpha} \int_{0}^{\tau} \mathbb{E}_{\alpha}\left(\frac{-\alpha}{1-\alpha}(\tau-\sigma)^{\alpha}\right) \eta(\sigma) d \sigma+\int_{0}^{\tau} \bar{f}(\sigma, \eta(\sigma)) d \sigma\right) \\
& \leq\left|\omega_{0}-\eta_{0}\right|+\frac{B(\alpha)}{1-\alpha} \int_{0}^{\tau} \mathbb{E}_{\alpha}\left(\left|\frac{-\alpha}{1-\alpha}(\tau-\sigma)^{\alpha}\right|\right)|\omega(\sigma)-\eta(\sigma)| d \sigma \\
& +\int_{0}^{\tau}|f(\sigma, \omega(\sigma))-\bar{f}(\sigma, \eta(\sigma))| d \sigma \\
& \leq\left|\omega_{0}-\eta_{0}\right|+\frac{B(\alpha)}{1-\alpha} \int_{0}^{\tau} \mathbb{E}_{\alpha}\left(\frac{\alpha}{1-\alpha}(\tau-\sigma)^{\alpha}\right)|\omega(\sigma)-\eta(\sigma)| d \sigma \\
& +\int_{0}^{\tau}|f(\sigma, \omega(\sigma))-f(\sigma, \eta(\sigma))| d \sigma+\int_{0}^{\tau}|f(\sigma, \omega(\sigma))-\bar{f}(\sigma, \eta(\sigma))| d \sigma \\
& \leq \epsilon_{1}+\frac{B(\alpha)}{1-\alpha} \int_{0}^{\tau} \mathbb{E}_{\alpha}\left(\frac{\alpha}{1-\alpha} T^{\alpha}\right)|\omega(\sigma)-\eta(\sigma)| d \sigma+\int_{0}^{\tau} p(\sigma)|\omega(\sigma)-\eta(\sigma)| d \sigma+\epsilon_{2} \int_{0}^{\tau} d \sigma \\
& \leq \epsilon_{1}+\epsilon_{2} T+\int_{0}^{\tau}\left[\frac{B(\alpha)}{1-\alpha} \mathbb{E}_{\alpha}\left(\frac{\alpha}{1-\alpha} T^{\alpha}\right)+p(\sigma)\right]|\omega(\sigma)-\eta(\sigma)| d \sigma .
\end{aligned}
$$

Applying Lemma 2.7, we get

$$
|\omega(\tau)-\eta(\tau)| \leq\left(\epsilon_{1}+\epsilon_{2} T\right) \exp \left(\int_{0}^{\tau}\left[\frac{B(\alpha)}{1-\alpha} \mathbb{E}_{\alpha}\left(\frac{\alpha}{1-\alpha} T^{\alpha}\right)+p(\sigma)\right] d \sigma\right), \tau \in J .
$$

From Eq. (5.4) we observe that the solution $\omega(\tau)$ of ABR-FDEs (1.1)-(1.2) depends continuously on the function involved the right side of Eq. (1.1).

Remark 5.3 Theorem 5.2 gives the dependency of the solution of the ABR-FDEs (1.1)(1.2) simultaneously on the initial condition and the functions involved on the right-hand side. If $\epsilon_{1}=0$ then it gives the dependency of solution on the function involved on right hand side, if $\epsilon_{2}=0$ then it gives the dependency of solution on initial condition and if $\epsilon_{1}=0$ and $\epsilon_{2}=0$ it gives the uniqueness of solution.

Let any $\delta, \delta_{0} \in \mathbb{R}$ and consider the following system of ABR-FDEs

$$
\frac{d \omega}{d \tau}+{ }_{0}^{*} \mathcal{D}_{\tau}^{\alpha} \omega(\tau)=g(\tau, \omega(\tau), \delta), \tau \in J, \omega(0)=\omega_{0} \in \mathbb{R},
$$




$$
\frac{d \omega}{d \tau}+{ }_{0}^{*} \mathcal{D}_{\tau}^{\alpha} \omega(\tau)=g\left(\tau, \omega(\tau), \delta_{0}\right), \tau \in J, \omega(0)=\omega_{0} \in \mathbb{R} .
$$

The following theorem shows dependency of solution of ABR-FDEs (5.5) and (5.6) on parameters.

Theorem 5.4 Let the function $g$ satisfies conditions of Theorem 4.3. Suppose there exists $p, q \in C\left(J, \mathbb{R}^{+}\right)$such that

$$
\begin{aligned}
|g(\tau, \omega, \delta)-g(\tau, \eta, \delta)| & \leq p(\tau)|\omega-\eta|, \\
\left|g(\tau, \omega, \delta)-g\left(\tau, \omega, \delta_{0}\right)\right| & \leq q(\tau)\left|\delta-\delta_{0}\right|,
\end{aligned}
$$

If $\omega_{1}, \omega_{2}$ are the solutions of $A B R-F D E s$ (5.5) and (5.6) respectively, then

$$
\left|\omega_{1}(\tau)-\omega_{2}(\tau)\right| \leq Q T\left|\delta-\delta_{0}\right| \exp \left(\int_{0}^{\tau}\left[\frac{B(\alpha)}{1-\alpha} \mathbb{E}_{\alpha}\left(\frac{\alpha}{1-\alpha} T^{\alpha}\right)+p(\sigma)\right] d \sigma\right), \tau \in J,
$$

where $Q=\sup _{\tau \in J} q(\tau)$.

Proof We find for any $\tau \in J$

$$
\begin{aligned}
\left|\omega_{1}(\tau)-\omega_{2}(\tau)\right| & \mid\left(\omega_{0}-\frac{B(\alpha)}{1-\alpha} \int_{0}^{\tau} \mathbb{E}_{\alpha}\left(\frac{-\alpha}{1-\alpha}(\tau-\sigma)^{\alpha}\right) \omega_{1}(\sigma) d \sigma+\int_{0}^{\tau} g\left(\sigma, \omega_{1}(\sigma), \delta\right) d \sigma\right) \\
& \quad-\left(\omega_{0}-\frac{B(\alpha)}{1-\alpha} \int_{0}^{\tau} \mathbb{E}_{\alpha}\left(\frac{-\alpha}{1-\alpha}(\tau-\sigma)^{\alpha}\right) \omega_{2}(\sigma) d \sigma+\int_{0}^{\tau} g\left(\sigma, \omega_{2}(\sigma), \delta_{0}\right) d \sigma\right) \mid \\
\leq & \frac{B(\alpha)}{1-\alpha}\left|\int_{0}^{\tau} \mathbb{E}_{\alpha}\left(\frac{-\alpha}{1-\alpha}(\tau-\sigma)^{\alpha}\right)\left(-\omega_{1}(\sigma)+\omega_{2}(\sigma)\right) d \sigma\right|+\int_{0}^{\tau}\left|g\left(\sigma, \omega_{1}(\sigma), \delta\right)-g\left(\sigma, \omega_{2}(\sigma), \delta_{0}\right)\right| d \sigma \\
\leq & \frac{B(\alpha)}{1-\alpha} \int_{0}^{\tau} \mathbb{E}_{\alpha}\left(\left|\frac{-\alpha}{1-\alpha}(\tau-\sigma)^{\alpha}\right|\right)\left|\omega_{1}(\sigma)-\omega_{2}(\sigma)\right| d \sigma+\int_{0}^{\tau}\left|g\left(\sigma, \omega_{1}(\sigma), \delta\right)-g\left(\sigma, \omega_{2}(\sigma), \delta\right)\right| d \sigma \\
& \quad+\int_{0}^{\tau}\left|g\left(\sigma, \omega_{2}(\sigma), \delta\right)-g\left(\sigma, \omega_{2}(\sigma), \delta_{0}\right)\right| d \sigma \\
\leq & \frac{B(\alpha)}{1-\alpha} \int_{0}^{\tau} \mathbb{E}_{\alpha}\left(\frac{\alpha}{1-\alpha}(\tau-\sigma)^{\alpha}\right)\left|\omega_{1}(\sigma)-\omega_{2}(\sigma)\right| d \sigma+\int_{0}^{\tau} p(\sigma)\left|\omega_{1}(\sigma)-\omega_{2}(\sigma)\right| d \sigma \\
& +\int_{0}^{\tau} q(\sigma)\left|\delta-\delta_{0}\right| d \sigma \\
\leq & \frac{B(\alpha)}{1-\alpha} \int_{0}^{\tau} \mathbb{E}_{\alpha}\left(\frac{\alpha}{1-\alpha} T^{\alpha}\right)\left|\omega_{1}(\sigma)-\omega_{2}(\sigma)\right| d \sigma+\int_{0}^{\tau} p(\sigma)\left|\omega_{1}(\sigma)-\omega_{2}(\sigma)\right| d \sigma+Q\left|\delta-\delta_{0}\right| \int_{0}^{\tau} d \sigma \\
\leq & \int_{0}^{\tau}\left[\frac{B(\alpha)}{1-\alpha} \mathbb{E}_{\alpha}\left(\frac{\alpha}{1-\alpha} T^{\alpha}\right)+p(\sigma)\right]\left|\omega_{1}(\sigma)-\omega_{2}(\sigma)\right| d \sigma+Q T\left|\delta-\delta_{0}\right| .
\end{aligned}
$$

Applying Lemma 2.7, we get

$$
\left|\omega_{1}(\tau)-\omega_{2}(\tau)\right| \leq Q T\left|\delta-\delta_{0}\right| \exp \left(\int_{0}^{\tau}\left[\frac{B(\alpha)}{1-\alpha} \mathbb{E}_{\alpha}\left(\frac{\alpha}{1-\alpha} T^{\alpha}\right)+p(\sigma)\right] d \sigma\right), \tau \in J
$$




\section{Example}

Consider a nonlinear ABR-FDEs of the form

$$
\begin{gathered}
\frac{d \omega}{d \tau}+{ }_{0}^{*} \mathcal{D}_{\tau}^{\frac{1}{2}} \omega(\tau)=f(\tau, \omega(\tau)), \tau \in J=[0,2], \\
\omega(0)=1,
\end{gathered}
$$

where $f: J \times \mathbb{R} \rightarrow \mathbb{R}$ is a continuous nonlinear function such that

$$
f(\tau, \omega(\tau))=\frac{|\omega(\tau)|+1}{2}+p(\tau), \tau \in J
$$

and

$$
p(\tau)=B\left(\frac{1}{2}\right)\left\{\tau \mathbb{E}_{\frac{1}{2}, 2}\left(-\tau^{\frac{1}{2}}\right)+\mathbb{E}_{\frac{1}{2}}\left(-\tau^{\frac{1}{2}}\right)-\tau-1\right\} .
$$

We observe that for any $\omega, \eta \in \mathbb{R}$ and for any $\tau \in J$,

$$
\begin{aligned}
|f(\tau, \omega)-f(\tau, \eta)| & =\left|\left(\frac{|\omega|+1}{2}+p(\tau)\right)-\left(\frac{|\eta|+1}{2}+p(\tau)\right)\right| \\
& =\frac{1}{2}|(|\omega|-|\eta|)| \leq \frac{1}{2}|\omega-\eta| .
\end{aligned}
$$

Thus the function $f$ satisfies Lipschitz condition with Lipschitz constant $L=\frac{1}{2}$. Compare with Theorem 4.3, we have $\alpha=\frac{1}{2}$ and $T=2$. Then the condition (4.4) reduces to

$$
8 \mathbb{E}_{\frac{1}{2}, 2}\left(2^{\frac{1}{2}}\right) B\left(\frac{1}{2}\right)<1
$$

This implies

$$
B\left(\frac{1}{2}\right)<\frac{1}{8 \mathbb{E}_{\frac{1}{2}, 2}\left(2^{\frac{1}{2}}\right)} .
$$

If we choose a normalizing function $B(\alpha)$ satisfying above condition, then by applying Theorem 4.3, ABR-FDEs (6.1)-(6.2) has unique solution. One can verify that ABR-FDEs (6.1)-(6.2) has the unique solution

$$
\omega(\tau)=\frac{\tau}{2}+1, \tau \in[0,2]
$$

\section{Conclusion}

Because of the presence of the nonsingular kernel in the equivalent fractional integral equation to FDEs involving $\mathrm{AB}$ derivatives, we can reasonably apply the Gronwall-Bellman inequality with continuous real valued functions to investigate the qualitative properties. Of Course, special attention needs in the case of complex valued functions. Further, 
one can acquire various qualitative properties of the higher class of fractional integrodifferential equations involving the $\mathrm{AB}$ fractional derivative in the sense of Caputo and Riemann-Liouville through the inequalities derived by B. G. Pachpatte [48]. But Pachpatte's inequalities can be applied only when the normalization function $B(\alpha)$ is strictly positive satisfying $B(0)=B(1)=1$.

\section{References}

1. Miller, K.S., Ross, B.: An introduction to the fractional calculus and fractional differential equations. John Wiley \& Sons, New York (1993)

2. Podlubny, I.: Fractional differential equations. Academic Press, San Diego (1999)

3. Lakshmikantham, V., Leela, S.: Theory of fractional dynamic systems. Cambridge Scientific Publishers, Cambridge (2009)

4. Kilbas, A.A., Srivastava, H.M., Trujillo, J.J.: Theory and applications of fractional differential equations, North-Holland Mathematics Studies, vol. 204. Elsevier Science, Amsterdam, The Netherlands (2006)

5. Diethelm, K.: The analysis of fractional differential equations. Springer-Verlag, Berlin (2010)

6. Diethelm, K., Freed, A. D.: On the solution of nonlinear fractional order differential equations used in the modeling of viscoplasticity, In: Scientific Computing in Chemical Engineering II- Computational Fluid Dynamics, Reaction Engineering and Molecular Properties, (F. Keil, W. Mackens, H. Voss, J. Werther (Eds)), pp 217-224, Springer-Verlag, Heidelberg, (1999)

7. Glockle, W.G., Nonnenmacher, T.F.: A fractional calculus approach of self-similar protein dynamics. Biophys. J . 68, 45-63 (1995)

8. Hilfer, R.: Application of Fractional Calculus in Physics. World Scientific, Singapore (2000)

9. Mainardi, F.: Fractional calculus: some basic problems in continuous and statistical mechanics, In: Fractals and Fractional calculus in Continuum Mechanics (A. Carpinteri and F. Mainardi (Eds.)), Fractals and Fractional Calculus in Continuum Mechanics, pp 291-348, Springer-Verlag, Wien and New York, (1997)

10. Metzler, F., Schick, W., Killian, H.G., Nonnenmacher, T.F.: Relaxation in filled polymers: A fractional calculus approach. J. Chem. Phys. 103, 7180-7186 (1995)

11. Tarasov, V.E.: Fractional Dynamics: Application of fractional calculus to dynamics of particles. Field and Media, Springer, New-York (2011)

12. Diethelm, K., Ford, N.J.: Analysis of fractional differential equations. J. Math. Anal. Appl. 265(2), 229-248 (2002)

13. Lakshmikantham, V.: Theory of fractional functional differential equations. Nonlinear Anal. 69, 33373343 (2008)

14. Lakshmikantham, V., Vatsala, A.S.: General uniqueness and monotone iterative technique for fractional differential equations. Appl. Math. Lett. 21, 828-834 (2008)

15. Agarwal, R.P., Zhou, Y., He, Y.: Existence of fractional neutral functional differential equations. Comput. Math. Appl. 59(3), 1095-1100 (2010)

16. Wang, J., Lv, L., Zhou, Y.: Ulam stability and data dependence for fractional differential equations with Caputo derivative. Elect. J. Qualit. Th Diff. Equ. 63, 1-10 (2011)

17. Caputo, M., Fabrizio, M.: A new definition of fractional derivative without singular kernel. Progr. Fract. Differ. Appl. 1(2), 73-85 (2015)

18. Atangana, A., Baleanu, D.: New fractional derivatives with nonlocal and non-singular kernel: Theory and application to heat transfer model. Therm. Sci. 20(2), 763-769 (2016)

19. Bonyah, E., Zarin Fatmawati, R.: Mathematical modeling of cancer and hepatitis co-dynamics with non-local and non-singular kernel, Commun. Math. Biol. Neurosci. 2020, Article ID 91, (2020)

20. Ahmad, S., Ullah, A., Akgul, A., Baleanu, D.: Analysis of the fractional tumour-immune-vitamins model with Mittag-Leffler kernel. Result. Phys. 19, 103559 (2020)

21. Kumar, S., Kumar, A., Samet, B., Gomez-Aguilar, J.F., Osman, M.S.: A chaos study of tumor and effector cells in fractional tumor-immune model for cancer treatment. Chaos, Solitons Fract. 141, $110321(2020)$

22. Ghanbari, B., Kumar, S., Kumar, R.: A study of behaviour for immune and tumor cells in immunogenetic tumour model with non-singular fractional derivative. Chaos, Solitons Fractals 133, 109619 (2020)

23. Thabet, S.T.M., Abdo, M.S., Shah, K., Abdeljawad, T.: Study of transmission dynamics of COVID-19 mathematical model under ABC fractional order derivative. Result Phys. 19, 103507 (2020) 
24. Logeswari, K., Ravichandran, C., Nisar, K. S.: Mathematical model for spreading of COVID-19 virus with the Mittag-Leffler kernel, Numer Methods Partial Differential Eq. (2020), 1-16

25. Jajarmi, A., Arshad, S., Baleuno, D.: A new fractional modeling and control strategy for the outbreak of dengue fever. Phys. A 535, 122524 (2019)

26. Baleanu, D., Jajarmi, A., Sajjadi, S.. S., Mozyrska, D.: A new fractional model and optimal control of a tumor-immune surveillance with non-singular derivative operator. Chaos Interdiscip J. Nonlinear Sci. 29(8), 083127 (2019)

27. Jajarmi, A., Ghanbari, B., Baleanu, D.: A new and efficient numerical method for the fractional modeling and optimal control of diabetes and tuberculosis co-existence. Chaos Interdiscip J. Nonlinear Sci. 29(9), 093111 (2019)

28. Jajarmi, A., Baleuno, D., Sajjadi, S.S., Asad, J.H.: A new features of the fractional Euler- Lagrange equation for a coupled oscillator using a nonsingular operator approach. Front. phys. 7, (2019). https:// doi.org/10.3389/fphy.2019.00196

29. Ucar, S., Ucar, E., Ozdemir, N., Hammouch, Z.: Mathematical analysis and numerical simulation for a smoking model with Atangana-Baleuno derivative. Chaos, Solitons Fract. 118, 300-306 (2019)

30. Abdo, M.S., Shah, K., Wahash, H.A., Panchal, S.K.: On comprehensive model of the novel coronavirus (COVID-19) under Mittag-Leffler derivative. Chaos, Solitons Fract. 135, 109867 (2020)

31. Jarad, F., Abdeljawad, T., Hammouch, Z.: On a class of ordinary differential equations in the frame of Atangana-Baleanu fractional derivative. Chaos, Solitons Fract. 117, 16-20 (2018)

32. Baleuno, D., Jajarmi, A., Hajipour, M.: On the nonlinear dynamical systems within the generalized fractinal derivative with Mittag-Leffler kernel. Nonlinear Dyn. 94, 397-414 (2018)

33. Syam, M.I., Al-Refai, M.: Fractional differential equations with Atangana-Baleanu fractional derivative: Analysis and applications. Chaos, Solitons Fract. 2, 100013 (2019)

34. Afshari, H., Baleanu, D.: Applications of some fixed point theorems for fractional differential equations with Mittag-Leffler kernel. Adv. Differ. Equ. 140, (2020)

35. Shah, K., Sher, M., Abdeljawad, T.: Study of evolution problem under Mittag-Leffler type fractional order derivative. Alexandria Eng. J. (2020). https://doi.org/10.1016/j.aej.2020.06.050

36. Ravichandran, C., Logeswari, K., Jarad, F.: New results on existence in the framework of AtanganaBaleanu derivative for fractional integro-differential equations. Chaos, Solitons Fract. 125, 194-200 (2019)

37. Ravichandran, C., Logeswari, K., Panda, S.K., Nisar, K.S.: On new approach of fractional derivative by Mittag-Leffler kernel to neutral integro-differential systems with impulsive conditions. Chaos, Solitons Fract. 139, 110012 (2020)

38. Kucche, K. D., Sutar, S. T.: Analysis of nonlinear fractional differential equations involving ABCaputo derivative, arXive:2007.09132

39. Sutar, S. T., Kucche, K. D.: On nonlinear hybrid fractional differential equations with AB-Caputo derivative, arXive:2007.11034

40. Mohamed, A.S., Mahmoud, R.A.: Picard, Adomian and perdictor-corrector methods for an initial value problem of arbitrary (fractional) prders differential equation. J. Egyptian Mathemat. Soc. 24, 165-170 (2016)

41. Kucche, K.D., Trujillo, J.J.: Theory of system of nonlinear fractional differential equations. Progr. Fract. Differ. Appl. 3, 7-18 (2017)

42. Pachpatte, B.G.: On certain Volterra integro-differential equations, FACTA UNIVERSITATIS. Ser. Math. Inform. 23, 1-12 (2008)

43. Kucche, K.D., Nieto, J.J., Venktesh, V.: Theory of nonlinear implicit fractional differential equations. Differ. Equ. Dyn. Syst. 28(1), 1-17 (2020)

44. Tidke, H.L.: Some theorems on fractional semiliear evolution equations. J. Appl. Anal. 18, 209-224 (2012)

45. Erdelyi, A., Magnus, W., Oberhettinger, F., Tricomi, F.G.: Higher Transcendental Functions, vol. I. McGraw-Hill, NewYork-Toronto-London (1953)

46. Kilbas, A.A., Saigo, M., Saxena, K.: Generalized Mittag-Leffler function and generalized fractional calculus operators. Integral Transforms Spec. Funct. 15, 31-49 (2004)

47. Prabhakar, T.R.: A singular integral equation with a generalized Mittag-Leffler function in the kernel. Yokohama. Math. J. 19, 7-15 (1971)

48. Pachpatte, B.G.: Inequalities for differential and integral equations, Mathematics in Science and Engineering Vol-197. Academic Press, San Diago (1998)

Publisher's Note Springer Nature remains neutral with regard to jurisdictional claims in published maps and institutional affiliations. 